\title{
Análisis de la eficacia publicitaria y usabilidad en herramientas Travel 2.0. Un estudio experimental a través de la técnica de eye-tracking
}

\section{Analysis of advertising effectiveness and usability in Travel 2.0 tools. An experimental study through eye-tracking technique}

\author{
Janet Hernández-Méndez \\ Universidad de Granada, Escuela de Doctorado de Humanidades y Ciencias Sociales y Jurídicas, Campus La Cartuja, s/n, 18071, Granada, España, \\ janethm23@gmail.com
}

\section{Francisco Muñoz-Leiva}

Universidad de Granada, Departamento de Comercialización e Investigación de Mercados, Facultad de Ciencias Económicas y Empresariales, Campus La Cartuja, s/n, 18071, Granada, España, franml@ugr.es

\section{Francisco J. Liébana-Cabanillas}

Universidad de Granada, Departamento de Comercialización e Investigación de Mercados, Facultad de Ciencias Económicas y Empresariales, Campus La Cartuja, s/n, 18071, Granada, España, franlieb@ugr.es

\section{Mauro Marchitto}

Universidad de Granada. Grupo de investigación Ergonomía y Ciencia Cognitiva, Instituto Mente, Cerebro y Comportamiento (CIMCYC), Campus La Cartuja, s/n, 18071, Granada, España, maurom@ugr.es

\begin{abstract}
Resumen
La finalidad del presente estudio es analizar el tipo de navegación que se realiza en las herramientas Travel 2.0 (blog de viajes, perfil en red social y en comunidad virtual de viajeros) así como la eficacia publicitaria de un banner ubicado en ellas. Para la consecución de estos objetivos se creó un diseño experimental con diferentes métricas oculares y un cuestionario autoadministrado que cada sujeto debía cumplimentar al término de cada prueba. Los resultados del estudio revelan lo siguiente: i) el patrón de visualización de los usuarios depende de la herramienta Travel 2.0 utilizada, ii) la no existencia de ceguera al banner comprobándose además que la edad y la experiencia afectan a la atención visual, y se demostró una mayor eficacia en el caso de Facebook y, por último, ii) las medidas de atención mediante eye-tracking y de recuerdo auto-informado difieren ligeramente entre sí.
\end{abstract}

Palabras clave: Banner, eye-tracking, ceguera al banner, recuerdo, Travel 2.0 tools.

\section{Abstract}

The goal of this study is to analyze the type of navigation used with Travel 2.0 tools (travel blogs, profiles on social networks and online travel communities), as well as the advertising efficacy of a banner on said sites. In order to achieve these objectives, an experimental design with different eye metrics and a self-administered questionnaire that each subject must complete at the end of each test. The results of this study reveal the following: i) the users' viewing patterns depend on the Travel 2.0 tool used, ii) the not existence of banner blindness in each Travel 2.0 tool, the age and experience affect to the visual attention and greater advertising efficacy was demonstrated in the case of Facebook and, finally, iii) the eye-tracking based attention measures differ slightly from measures of self-reported memory.

Keywords: Banner, eye-tracking, banner blindness, memory, Travel 2.0 tools.

\section{Introducción}

En los últimos años los medios de comunicación han cambiado considerablemente y como consecuencia ha aparecido un nuevo mundo online de colaboración y comunicación: la Web 2.0 (Cheung, Chiu, \& Lee, 2011). El consumidor de hoy en día está más conectado que nunca, con más accesos y con una participación más activa y profunda en el contenido (Nielsen, 2014). Este desarrollo de la Web ha afectado por un lado, a los distintos sectores de actividad, entre los que se encuentra el sector turístico, dando lugar a las denominadas herramientas Travel 2.0 (Mendes-Filho \& Tan, 2008; Muñoz-Leiva, Hernández-Méndez, \& Sánchez-Fernández, 2012; Hernández-Méndez, Muñoz-Leiva \& Sánchez-Fernández, 2015). Estas herramientas (blogs de viajes, redes sociales de viajes, foros, etc.) permiten al turista adoptar un papel más activo a la hora de decidir y preparar sus viajes, y además ayudar al resto de viajeros a formarse una imagen previa del destino que van a visitar (Muñoz-Leiva et al., 2012). Por tanto, las empresas turísticas necesitan integrar este tipo de herramientas, cuyo contenido es generado por los usuarios, en sus propias estrategias de marketing (Öz, 2014).
Por otro lado, es evidente que el entorno de los medios en los que se inserta la publicidad está cambiando (Chiou, Wan, \& Lee, 2008) y en este sentido podemos encontrar diferentes formatos publicitarios utilizados también en estas herramientas Web 2.0, tales como banners, vídeos promocionales, enlaces patrocinados, etc. El tipo de publicidad online más antigua y utilizada ha sido el banner. El primer banner apareció en la página www.hotwired.com en Octubre de 1994 (Hollis, 2005; Abuín, 2008) y como consecuencia surgió un nuevo "Reino" de la publicidad (Lohtia, Donthu, \& Yaveroglu, 2007). Desde entonces los anunciantes no han parado de destinar recursos y esfuerzos a crear banners que no sólo llamen la atención del usuario sino que además le transmitan confianza (Gong \& Maddox, 2003). Una vez captada su atención, el objetivo es que el internauta haga clic sobre el anuncio y sea dirigido a la web del anunciante (Abuín, 2008). A su vez ante esta creciente saturación publicitaria aparece el concepto de "banner blindness" que provoca una reducción de la proporción de clic que se realizan sobre un banner (click-through rate, CTR). Ante esta problemática, los investigadores analizan 
cuál es el motivo que da lugar a la ceguera hacia la publicidad online (tipo de banner, contenido del anuncio, etc.) y los anunciantes buscan dar respuesta a cómo mejorar la eficacia publicitaria (Hernández-Méndez \& Muñoz-Leiva, 2015).

El presente trabajo tiene por finalidad evaluar la eficacia publicitaria en tres herramientas Travel 2.0 concretas: un blog de viajes, red social y comunidad virtual con contenido turístico, a través de la medición de variables psicofisiológicas de atención y procesamiento cognitivo, junto a medidas post hoc de recuerdo espontáneo y guiado o sugerido. En particular se propusieron diferentes objetivos más específicos como: (i) identificar qué tipo de atención visual desarrollan los usuarios cuando visitan estas herramientas, (ii) comprobar si existe ceguera al banner, y en qué sujetos y herramientas se produce en mayor medida, (iii) determinar el recuerdo de los usuarios hacia el banner. Para ello se llevará a cabo un experimento en el que se utilizará la técnica o metodología de eye-tracking así como un cuestionario autoadministrado aplicado a un total de 60 participantes.

A partir de los objetivos de investigación planteados en este epígrafe, realizaremos una revisión teórica sobre la importancia del eye-tracking, la ceguera y el recuerdo del banner en el siguiente epígrafe. En el tercer epígrafe se plantean las cuestiones metodológicas relacionadas con el experimento llevado a cabo así como se detalla el ámbito de estudio y el proceso de recogida de información para dar respuesta a los objetivos. A continuación se extraen y describen las principales conclusiones e implicaciones para la gestión.

\section{Revisión de la literatura}

\subsection{La técnica de eye-tracking para la medición de la atención}

Gran parte de las herramientas que miden el grado de atención que muestran los consumidores a un conjunto de estímulos basadas en el auto-reporte siempre ha sido una tarea problemática y fuente de limitaciones en los estudios de investigación. Para solucionar estos problemas surgen nuevas técnicas o metodologías procedentes de las neurociencias y la psicología como el eye-tracking. Las aplicaciones de estas metodologías abren un sinfín de nuevas posibilidades para el estudio de la atención hacia la publicidad online y el marketing en general.

Los consumidores mueven sus ojos con el objetivo de obtener información y se detienen en aquello que les llama la atención. EI ojo es un órgano tremendamente complejo capaz de moverse a gran velocidad y la capacidad de recoger los movimientos rápidos de los ojos (saccades o movimientos sacádicos) y las fijaciones implica una opción muy interesante para estudiar el procesamiento de la información por parte de los individuos (Russo, 1978). Las fijaciones se producen cuando el ojo se estabiliza con una duración de entre 200 a $300 \mathrm{msec}$ (Pan \& Zhang, 2010), o entre 200 y 500 msec en tareas de lectura (Rayner, 1998).

El "scanpath" es el patrón que sigue el ojo atendiendo a sus movimientos (saccades y fijaciones), a través de estímulos visuales (Noton \& Stark, 1971). Estos caminos que el ojo sigue y las fijaciones son captados por los sistemas de eye-tracking mediante la captación de la reflexión de un haz de luz infrarroja en el centro de la pupila (reflexión corneal infrarroja). Su estudio puede aplicarse para el diseño de estímulos implicados en el marketing visual (bottom-upfactors) que persigan captar la atención de los consumidores así como conocer cómo influyen las características propias del individuo (top-downfactors) en el proceso voluntario de atención.

En este contexto, Wedel and Pieters (2008) encuentran en la literatura científica una relativa escasez de aplicaciones basadas en la metodología de eye-tracking que examinen los posibles efectos de los estímulos visuales de marketing y sus efectos en los consumidores. Esta escasez puede deberse a tres creencias principales (Wedel \& Pieters, 2008): 1) la atención es una mera pre-condición, 2) la captación y la retención de la atención son consideradas como reacciones relativamente simples y, 3) la utilización de estas metodologías resulta bastante complicada.

Por último, la problemática de medir la atención hacia las exposiciones a los estímulos de marketing visual, hizo que sus mediciones se aproximaran a través de otras como el recuerdo o respuesta emocional manifestada o haciendo uso de la memoria. Sin embargo, en muchas ocasiones la atención puede producirse a un nivel bajo de conciencia, es decir, la información puede ser activamente procesada por la existencia de información externa o representaciones internas de conocimiento ya presentes en la memoria. Esto pondría en entredicho las medidas de recuerdo y el reconocimiento tradicionalmente empleadas (Van Trijp, 2009). En nuestro caso, los sistemas de eye-tracking ofrecen una respuesta a esta problemática y podremos comparar los resultados de la atención con el recuerdo auto-reportado por los sujetos.

\subsection{Qué miran y qué patrón de visualización siguen los usuarios cuando entran a un sitio web}

Con respecto al primer objetivo de investigación, los estudios realizados con la técnica de eye-tracking suelen modelizar la ruta de la visión del usuario (orientación, primeras fijaciones, cuántas regiones de interés recorren o son más frecuentes,...).

Lindgaard, Fernandes, Dudek, and Brown (2006) realizaron tres estudios para determinar cuánto tiempo tardan los usuarios en formarse una opinión sobre el atractivo visual de una página web, y descubrieron que los internautas pueden construir una decisión fiable, sobre si les gusta o no la página, en 50 milisegundos. Por tanto, los diseñadores web disponen de este tiempo para crear una buena impresión sobre la página web.

Tras una profunda revisión de la literatura (ver síntesis en tabla 1) sobre los diferentes patrones de visualización en las páginas web podemos concluir que los usuarios se fijan principalmente en la parte superior y en el centro de la página, y además que el movimiento de los ojos normalmente va desde el lado izquierdo al lado derecho de la misma. En otras palabras, la parte inferior de la web y el lado derecho son las áreas que menos fijaciones suelen recibir. Sin embargo, esto puede variar en función del tipo de página web o la tarea asignada al sujeto. 
Tabla 1 - Revisión de la literatura sobre los patrones de visualización

\begin{tabular}{|c|c|}
\hline $\begin{array}{l}\text { Dirección del patrón de } \\
\text { visualización }\end{array}$ & Principales resultados \\
\hline \multirow{2}{*}{$\begin{array}{l}\text { Del centro-lado izquierdo al } \\
\text { lado derecho de la página } \\
\text { web }\end{array}$} & $\begin{array}{l}\text {-Los usuarios primero miran el centro de la web, después el lado izquierdo y por } \\
\text { último el lado derecho. } \\
\text {-Los usuarios apenas se fijan en la parte inferior de la pantalla que es visible. }\end{array}$ \\
\hline & $\begin{array}{l}\text {-Los usuarios se centran en el medio y en la parte superior de la página web. } \\
\text {-Los usuarios miran los logos en sus primeras fijaciones. }\end{array}$ \\
\hline \multirow{3}{*}{ De izquierda a derecha } & $\begin{array}{l}\text {-Los usuarios principalmente se fijan en la parte superior izquierda de la página. } \\
\text { Después el movimiento de los ojos va de izquierda a derecha y por último, } \\
\text { desplazan su mirada a la parte inferior de la pantalla. }\end{array}$ \\
\hline & $\begin{array}{l}\text {-Los usuarios tienen un patrón de visualización de izquierda a derecha, ya que los } \\
\text { participantes completaron la tarea que requiere información del lado izquierdo } \\
\text { más rápido que la que requiere información del lado derecho. }\end{array}$ \\
\hline & $\begin{array}{l}\text {-Los participantes se centraron principalmente en el menú principal situado en el } \\
\text { lado izquierdo de la página web y luego buscaron por el contenido. }\end{array}$ \\
\hline \multirow{2}{*}{ Patrón de visualización "F" } & $\begin{array}{l}\text {-Primero los usuarios leen la parte superior del contenido con un movimiento } \\
\text { horizontal, formando la línea superior de la "F". } \\
\text {-Luego, los usuarios se mueven un poco más hacia abajo y leen a través de un } \\
\text { segundo movimiento horizontal que normalmente cubre un área más corta que el } \\
\text { movimiento anterior. } \\
\text {-Por último, los usuarios visualizan el lado izquierdo del contenido en un } \\
\text { movimiento vertical. }\end{array}$ \\
\hline & $\begin{array}{l}\text {-El patrón visual en forma de " } \mathrm{F} \text { " no se cumple en el caso de páginas web basadas } \\
\text { en imágenes. En este caso los participantes centraban su mirada principalmente } \\
\text { en las fotos que estaban encima del pliegue (parte de la página web visible sin } \\
\text { necesidad de desplazar la barra lateral hacia abajo). }\end{array}$ \\
\hline $\begin{array}{l}\text { Patrones de visualización } \\
\text { según } \\
\text { ciertos factores }\end{array}$ & Principales resultados \\
\hline Tarea del usuario & $\begin{array}{l}\text {-Los resultados del estudio muestran que cuando los usuarios de Internet navegan } \\
\text { por una página web, se fijan en mayor medida en su parte central. } \\
\text {-Sin embargo, cuando los usuarios buscan una información específica, le prestan } \\
\text { más atención al lado izquierdo. }\end{array}$ \\
\hline \multirow{2}{*}{ Contenido página web } & $\begin{array}{l}\text {-Al visualizar una página web los usuarios se fijaban en mayor medida en las } \\
\text { imágenes principales, en las caras de los famosos y en la función del buscador. Así } \\
\text { mismo aquellas partes de la web que tenían mucho texto recibían menos } \\
\text { fijaciones. }\end{array}$ \\
\hline & $\begin{array}{l}\text {-Las páginas web con imágenes de personas no sólo son percibidas como más } \\
\text { atractivas sino que además permite a los usuarios completar la tarea de forma más } \\
\text { rápida. }\end{array}$ \\
\hline
\end{tabular}

\begin{tabular}{|l} 
Autores \\
Namahn (2001) \\
\hline Djamasbi, Siegel, \\
and Tullis (2010a) \\
Castellucio (2004) \\
\hline $\begin{array}{l}\text { Djamasbi, Siegel, Tullis, } \\
\text { and Dai (2010b) }\end{array}$ \\
\hline
\end{tabular}

Mazman, Akbal, Tüzün, and Yeniad (2010)

Nielsen (2006)

Shrestha and Lenz (2007)

Autores

Djamasbi , Siegel, and Tullis (2011)

Djamasbi et al. (2010a)

Djamasbi et al. (2010b)

Fuente: Elaboración propia.

Particularmente, en nuestro estudio daremos una respuesta a la siguiente pregunta: ¿Cuál es el patrón de visualización de los usuarios de blogs de viajes, redes sociales de viajes y comunidades virtuales de viajeros?

\subsection{Ceguera al banner}

\subsubsection{Causas y consecuencias de la ceguera al banner}

Con respecto al segundo objetivo propuesto acerca de la eficacia del banner en términos de atención, la revisión de la literatura pone de manifiesto que existen muchos usuarios que no recuerdan los banners durante su visita en la página web, y además existen usuarios que los evitan (e.g. Bayles, 2002; Nielsen, 2007).

Los usuarios no sólo aprenden rápidamente la estructura de la página web sino que además utilizan experiencias de navegación pasadas para evitar los banners y así centrarse en el contenido principal (Hsieh \& Chen, 2011). Esto se conoce con la expresión 'ceguera a los banners' que hace alusión a que los usuarios los ignoran y/o no prestan atención a su contenido (Burke, Hornof, Nilsen, and Gorman, 2005; Margarida, 2013). Con respecto a esto, la publicidad online que distrae a los usuarios del contenido de la página web puede ser considerada como intrusiva y generar una percepción negativa en los internautas, no sólo hacia los productos y servicios que anuncian, sino también hacia la marca y el medio, como puede ser la página web (Abuín, 2008).

La mayoría de las fijaciones sobre los banners se producen en los primeros movimientos oculares, para así poder evitarlos durante la visita (Burke et al., 2005). Así mismo la visión periférica permite a los usuarios realizar un repaso superficial de la página web en la que se encuentran, y puesto que los anuncios tienen un formato gráfico que difiere del contenido editorial pueden filtrarse rápidamente (León, 2009). A continuación (tabla 2) se presentan los principales resultados de la revisión de la literatura en relación a la existencia de ceguera hacia los banners. 
Tabla 2 - Revisión de la literatura sobre ceguera al banner

\begin{tabular}{|c|c|c|}
\hline Factores & Principales resultados & Autores \\
\hline \multirow{3}{*}{$\begin{array}{l}\text { Contenido } \\
\text { editorial vs. } \\
\text { banner }\end{array}$} & $\begin{array}{l}\text { - Los usuarios no se fijan en los elementos con un diseño similar a un anuncio, incluso si éstos no tienen } \\
\text { propósito comercial. }\end{array}$ & Nielsen (2007) \\
\hline & $\begin{array}{l}\text { - Si se integra la publicidad en el contenido de la página, se impide que los usuarios identifiquen } \\
\text { rápidamente los anuncios y los descarten como información a consultar. }\end{array}$ & Abuín (2008) \\
\hline & $\begin{array}{l}\text { - En Facebook los sujetos le prestan más atención a las recomendaciones de amigos en Facebook, que a } \\
\text { los banners de esta red social. } \\
\text { - Por tanto, en las redes sociales los anuncios también son ignorados, y existe ceguera al banner }\end{array}$ & Margarida (2013) \\
\hline \multirow{3}{*}{$\begin{array}{l}\text { La posición } \\
\text { del banner }\end{array}$} & $\begin{array}{l}\text { - En este estudio la mayoría de participantes nunca se fijan en el anuncio, y los que sí se fijan lo hacen por } \\
\text { un periodo corto de tiempo. }\end{array}$ & $\begin{array}{l}\text { eVOC Insights } \\
\text { (2004) }\end{array}$ \\
\hline & $\begin{array}{l}\text { - La ceguera a los banners es más frecuente cuando el anuncio se encuentra en el lado derecho de la página } \\
\text { que en la parte superior }\end{array}$ & $\begin{array}{l}\text { Owens, Chaparro, } \\
\text { and Palmer (2011) }\end{array}$ \\
\hline & $\begin{array}{l}\text { - Para aumentar la fijación en el banner de los periódicos online sería recomendable colocarlo muy próximo } \\
\text { al texto principal de la noticia o incluso dentro de ésta. }\end{array}$ & $\begin{array}{l}\text { Mosconi, Porta, and } \\
\text { Ravarelli (2008) }\end{array}$ \\
\hline \multirow[t]{2}{*}{$\begin{array}{c}\text { Tipos de } \\
\text { tareas }\end{array}$} & $\begin{array}{l}\text { - Cuando los usuarios realizan búsquedas sencillas en el medio online no necesitan prestarle tanta atención } \\
\text { y por tanto pueden percibir y procesar otros estímulos. } \\
\text { - Sin embargo las búsquedas con un mayor grado de dificultad requieren más atención, lo que da lugar a } \\
\text { que los usuarios dispongan de menos tiempo para procesar objetos irrelevantes, y por tanto los ignoren. }\end{array}$ & Burke et al. (2005) \\
\hline & $\begin{array}{l}\text { - Sólo los anuncios que están muy relacionados con la finalidad del sujeto podrán conseguir resultados } \\
\text { positivos, ya que el usuario evitará todo el contenido que no corresponda con su propósito. }\end{array}$ & León (2009) \\
\hline
\end{tabular}

Fuente: Elaboración propia.

La revisión de la literatura nos muestra resultados, en algunos casos, contradictorios o dependientes del tipo de sitio web. Por tanto, en nuestra investigación planteamos si existe ceguera a los banners según el tipo de herramienta Travel 2.0: blogs de viajes, redes sociales de viajes y comunidades online de viajeros. Adicionalmente, nos planteamos si esta ceguera depende de las características del sujeto como pasamos a comentar a continuación.

\subsubsection{Ceguera al banner según variables de clasificación del usuario}

En nuestro estudio también queremos explorar cómo influyen determinadas variables demográficas (género y edad) y la experiencia (medida como la frecuencia de uso) en la ceguera al banner de estos nuevos sistemas.

\section{Género}

En primer lugar, en la literatura científica encontramos pistas acerca de que el comportamiento de visualización hacia una página web varía según el género del usuario. Según San José, Gutiérrez, and Gutiérrez (2004) los hombres son más propensos que las mujeres a contactar con la empresa anunciada y a comprar su producto anunciado. Asimismo los resultados obtenidos por Goodrich (2014) muestran que los hombres le prestan más atención a los banners que las mujeres.

Sin embargo, otros autores afirman que no existen diferencias significativas en el género con respecto a los anuncios online, y por tanto, hombres y mujeres se comportan prácticamente de la misma manera (e.g. Drèze \& Hussherr, 2003; Margarida, 2013).

\section{Edad}

Las personas mayores miran el mismo número de zonas que los jóvenes, pero se fijan en un mayor número de áreas produciéndose así una mayor duración de la fijación (Drèze \& Hussherr, 2003). Aunque, Danaher, Mullarkey, and Essegaier (2006) afirman que la publicidad web sería menos las personas jóvenes que para los mayores, ya que éstos permanecen más tiempo en sitios con anuncios.

\section{Experiencia}

Se ha comprobado que los usuarios que tienen menos experiencia en Internet son más propensos a hacer clic en los banners que los usuarios que tienen más experiencia (Dahlen, 2001). A diferencia de este estudio citado, Thorbjørnsen, Supphellen, Nysveen, and Egil (2002) comprobaron que el usuario novel será menos propenso a interactuar con la publicidad, ya que le presta una mayor atención a elementos complementarios al anuncio como es el entorno web. Por tanto, se ha demostrado que los usuarios con más experiencia en Internet se exponen de forma más activa al banner promocional que los noveles (Crespo 2011).

\subsection{Recuerdo de los banners}

La publicidad efectiva es aquella que no sólo capta la atención del público, sino la que también permanece en su memoria, ya sea a corto o a largo plazo (Margarida, 2013). En este sentido, a los anunciantes no sólo les importa que se le preste atención a los anuncios, sino que también se recuerde. Tras revisar la literatura relevante en esta materia se ha comprobado que muchos de los anuncios no son recordados por los participantes en los estudios de investigación (Bayles, 2002; Drèze \& Hussherr, 2003; Burke et al., 2005).

Yaveroglu and Donthu (2008) analizaron los efectos de la repetición del banner en el recuerdo de la marca y en la intención de hacer clic. Se concluyó que la repetición de la publicidad crea mayor recuerdo y mayor intención de hacer clic en el entorno online. Para Crespo (2011) lo que más se recuerda del banner es la marca anunciada. De forma adicional, los resultados de Gong and Maddox (2003) sugieren que sólo una exposición adicional del banner mejora el recuerdo hacia la marca.

Los resultados del trabajo de Drèze and Hussher (2003) demostraron que el $46,9 \%$ de los sujetos indicaron que recordaban haber visto algún banner en la página web de las cuatro opciones mostradas (dos banner reales y dos ficticios); sin saber diferenciar entre los reales y los ficticios. 
Por tanto, nuestro último objetivo busca dar respuesta a la pregunta: ¿̇los usuarios de blogs de viajes, redes sociales de viajes y comunidades de viajeros son capaces de recordar los anuncios a los que han sido expuestos?

\section{Detalles del experimento}

\subsection{Trabajo de campo y sistema de medición utilizado}

El trabajo de campo se llevó a cabo del 15 al 22 de Noviembre de 2013 en el Centro de Investigación, Mente, Cerebro y Comportamiento (Mind, Brain and Behavior Research Center CIMCYC-) de la Universidad de Granada. El diseño experimental propuesto pretendía conseguir un total de 60 pruebas válidas, con edades comprendidas entre los 16 y 57 años. El procedimiento de muestreo estuvo basado en un método de "bola de nieve" y se contactó con los sujetos potenciales por email y teléfono, ofreciendo un incentivo de $15 €$ por participar en el estudio.

El estudio de laboratorio consistió en la entrada del sujeto en una habitación tranquila, aislada de cualquier ruido exterior, con una luz ambiental de 200 lúmenes, tal y como recomienda la International Telecommunication Union (2002) para simular un ambiente familiar ('home environment'). En particular, el sistema de seguimiento ocular usado fue el TobiiT60 de $60 \mathrm{~Hz}$ (figura 1) con un nivel de precisión de $0,5^{\circ}$ integrado en un monitor TFT de 17 " con una resolución de pantalla de 1280 x 1024 píxeles.

Figura 1 - Sistema de eye-tracking utilizado

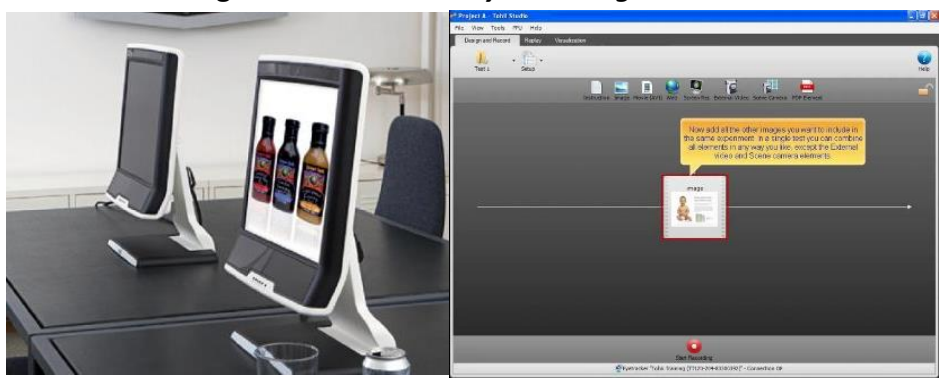

Fuente: http://www.tobii.com.

\subsection{Diseño experimental}

El diseño experimental inter-grupo (comparativa por grupos de sujetos) e intra-sujetos (comparativa entre herramientas analizadas) estuvo basado en una réplica de las tres herramientas Travel 2.0 del Hotel Jardín Tropical (situado en Adeje-Tenerife, España): su blog, un perfil en la red social Facebook y en la comunidad virtual, como estudios previos han utilizado (e.g. Muñoz-Leiva et al., 2012; Margarida, 2013) o justificado (e.g. Buhalis \& Law, 2008; Muñoz-Leiva et al., 2012). La única similitud con respecto a los sitios originales fue la de incrustar en cada herramienta un banner de una compañía aérea (Air Europa) con tres personales famosos (figura 2). Este banner contenía texto ("Volamos solo para ti! Entra en" + URL + "y ganas premios todas las semanas") y una imagen compuesta por personas y un avión. Este diseño se basó en diferentes medidas de parámetros oculares considerando diferentes áreas de interés. A continuación aparece el área de interés fundamental en nuestro estudio de investigación: el banner publicitario.

Figura 2 - Banner para la aerolínea usada en el experimento

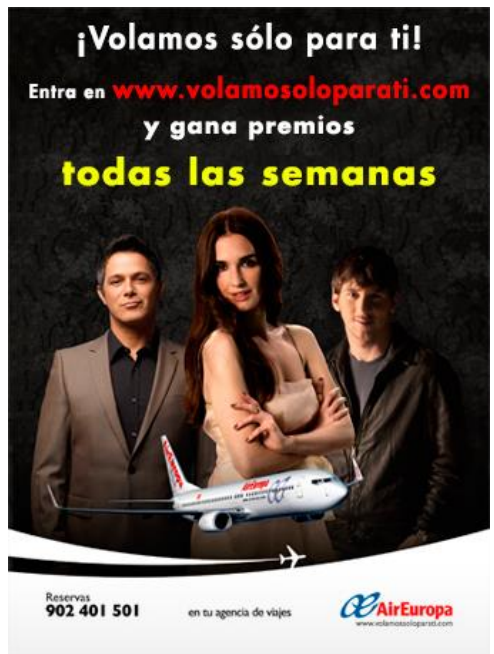

Por tanto, el diseño final consistió en la presentación repetida distintos de las siguientes herramientas Travel 2.0 (escenarios experimentales):

X1: Blog del hotel con el banner de la aerolínea

(url: http://webcim.ugr.es/polls/EP_ET/X1.html); 90 segundos

X2: Perfil del hotel en Facebook con el banner de la aerolínea.

url: http://webcim.ugr.es/polls/EP_ET/X2.html); 90 segundos

X3: Perfil del hotel en Tripadvisor con el banner de la aerolínea.

(url: http://webcim.ugr.es/polls/EP_ET/X3.html); 90 segundos

Se eligieron tres órdenes diferentes de presentación de los escenarios experimentales para mitigar el posible efecto debido a este orden de presentación. Además, en nuestro caso el diseño estuvo balanceado para los diferentes rangos de edad (mediana = 35 años para formar grupos de igual tamaño) y género. Además se garantizó la asignación aleatoria de los sujetos a cada grupo experimental (Malhotra, 1997). La aleatoriedad permite un reparto equilibrado de los efectos de las variables independientes bajo las mismas condiciones y asegura que el número total de repeticiones del experimento mostrará los verdaderos efectos, si ellos existen realmente (Zikmund, 2003).

De esta forma garantizamos una adecuada validez interna, consecuencia de poder ejercer un mayor control del efecto de las variables independientes y del escenario de investigación (Zikmund, 2003). Sin embargo, podría introducirse errores originados por el carácter artificial del entorno, lo que provocaría una baja validez ecológica de los resultados. Por este motivo se consideraron tres herramientas Travel 2.0 distintas que permiten aumentar las posibilidades de generalización de los resultados al conjunto de dichas herramientas y con ello la validez externa de los resultados. 
Con respecto al proceso de recogida de datos, el sujeto tras firmar el documento de consentimiento informado y el recibí del incentivo, se sentó frente al ordenador y se le explicó la tarea a realizar consistente en buscar información en forma de texto integrado en el contenido editorial que versa sobre las vistas que ofrecían las habitaciones del hotel. A través de este mecanismo se pretende conseguir cierto grado de implicación con el experimento y una navegación orientada a una tarea al igual que podría ocurrir en una situación normal dentro de este tipo de herramientas Travel 2.0. Tras el proceso de calibración de su mirada, el sujeto pasa automáticamente a una inspección de las tres herramientas web durante un tiempo total de cuatro minutos y medio (90 segundos por cada herramienta). Por último, el participante pasó a otro ordenador ubicado fuera de la sala con el eye-tracker, donde realizó un cuestionario autoadministrado en el que tenía que responder a diferentes preguntas de clasificación y otras relativas al recuerdo del banner (espontáneo y sugerido).

\section{Análisis de resultados}

\subsection{Patrón de visualización en herramientas Travel 2.0}

En primer lugar, comprobamos cuál es el patrón de movimiento y fijación ocular de los participantes con ayuda de los gráficos de fijación de la mirada (gazeplots) y los mapas de calor (heatmaps) que nos proporciona el software Tobii Studio.

\subsubsection{Blog}

Un análisis detallado de los patrones de visualización más frecuentes en el caso del blog (figura 3), muestra que los participantes comienzan visualizando el primer post formado por una imagen donde se contempla a una chica tumbada en una hamaca, y por la parte del texto correspondiente a esta primera publicación. A continuación los sujetos dirigieron su mirada a la cabecera del blog donde se encuentra el logo del hotel y una foto de las instalaciones del mismo.

Figura 3 - Gazeplot del sujeto más representativo y heatmap del blog del hotel

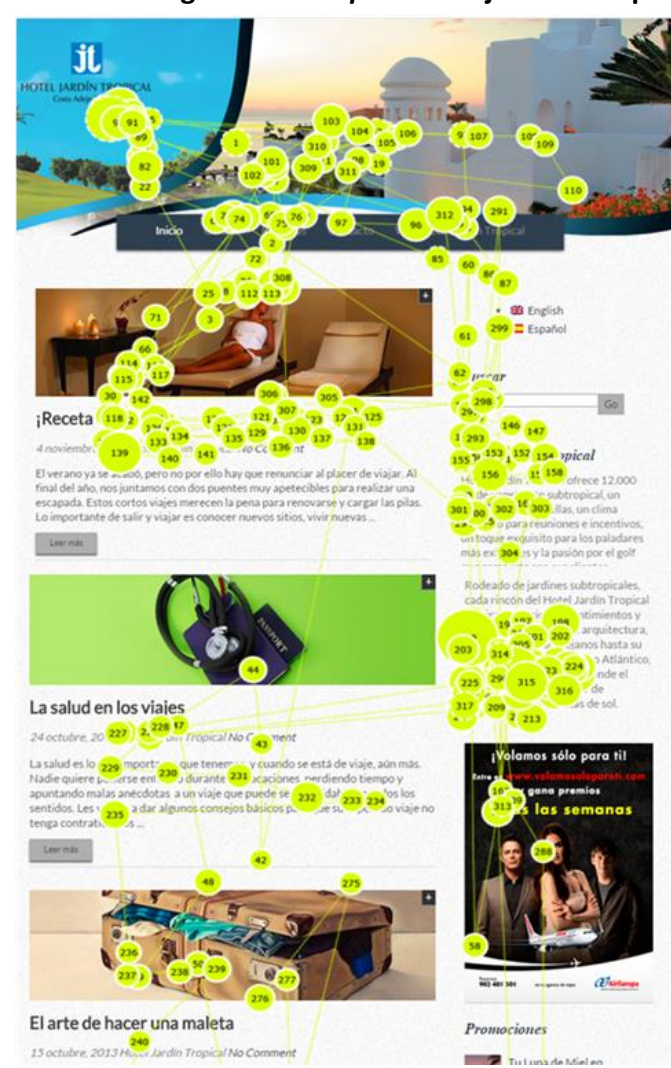

Después de visualizar el área superior de la página, los usuarios siguieron un patrón de visualización de izquierda a derecha, probablemente para leer el texto disponible en la página web y así poder encontrar la información referente a la tarea. Así mismo en este recorrido se comprueba que algunos participantes se fijaron en el banner objeto de estudio.

Hay que destacar que, en contra de lo que sostienen o descubren estudios anteriores, en el blog los usuarios también se fijan en la parte inferior de la página, especialmente en el lado derecho, donde se encuentra la galería de fotos del mismo. Probablementeesto puede deberse a que los usuarios pensaban que el resultado de la tarea se encontraba en esas fotos.

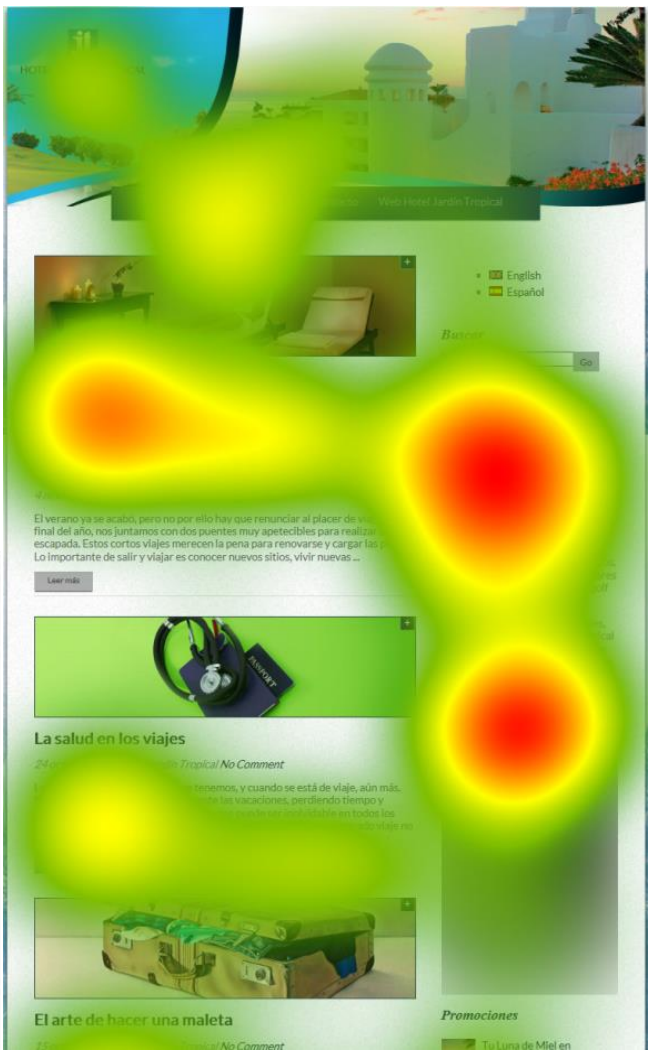

Según el heatmap, las zonas que reúnen un mayor número de fijaciones (zonas más calientes) por parte de los usuarios son: la descripción del hotel situada a la derecha de la página, la parte superior del banner objeto de estudio, el comentario de un cliente situado en la parte inferior de la pantalla (donde se encuentra el resultado de la tarea) y el contenido del primer post.

\subsubsection{Facebook}

Cuando los usuarios visualizaron la página de Facebook del hotel seleccionado (ver figura 4), primero se fijaron en la parte superior izquierda de la pantalla, donde se encontraba la foto de portada y la del perfil de la página. Concretamente estas dos fotos son las que más atención reciben. 
Figura 4 - Gazeplot del sujeto más representativo y heatmap del perfil en Facebook del hotel

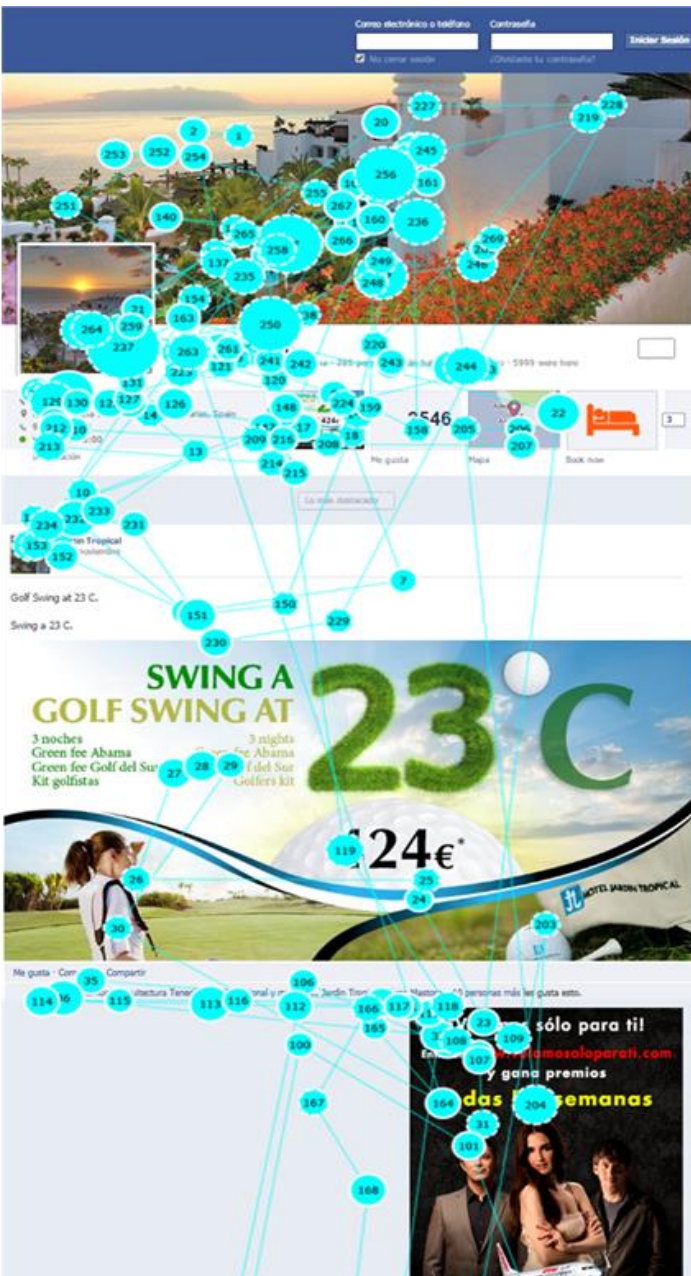

Después de visualizar esta área durante bastante tiempo, los participantes pasaban a explorar la primera publicación de la página referente a una oferta de campo de golf, aunque en este caso las fijaciones tenían una menor duración. Posteriormente se fijaban en el banner, ubicado justo debajo de la primera publicación y donde las fijaciones también fueron más cortas. En tercer lugar, se le prestaba atención a la última publicación de la página en la que se mostraba una foto del hotel. En este caso los usuarios detuvieron su mirada durante más tiempo e incluso leyeron el contenido situado debajo la foto, ya que en ese mensaje se encontraba la información necesaria para la tarea que se había encomendado a los usuarios al principio del experimento.

A modo de resumen podemos decir por un lado, que el patrón de visualización de los usuarios de Facebook va de arriba hacia abajo, y por otro lado, que la parte superior izquierda de la pantalla y la publicación que contiene la información necesaria para completar la tarea del experimento son las áreas a las que los usuarios le prestan más atención.

\subsubsection{Tripadvisor}

Al igual que en el caso anterior, en la página de Tripadvisor los usuarios primero se fijan en la parte superior izquierda de la pantalla donde se encuentra el nombre el hotel y las fotos, tanto profesionales como las subidas por los propios viajeros. El sistema

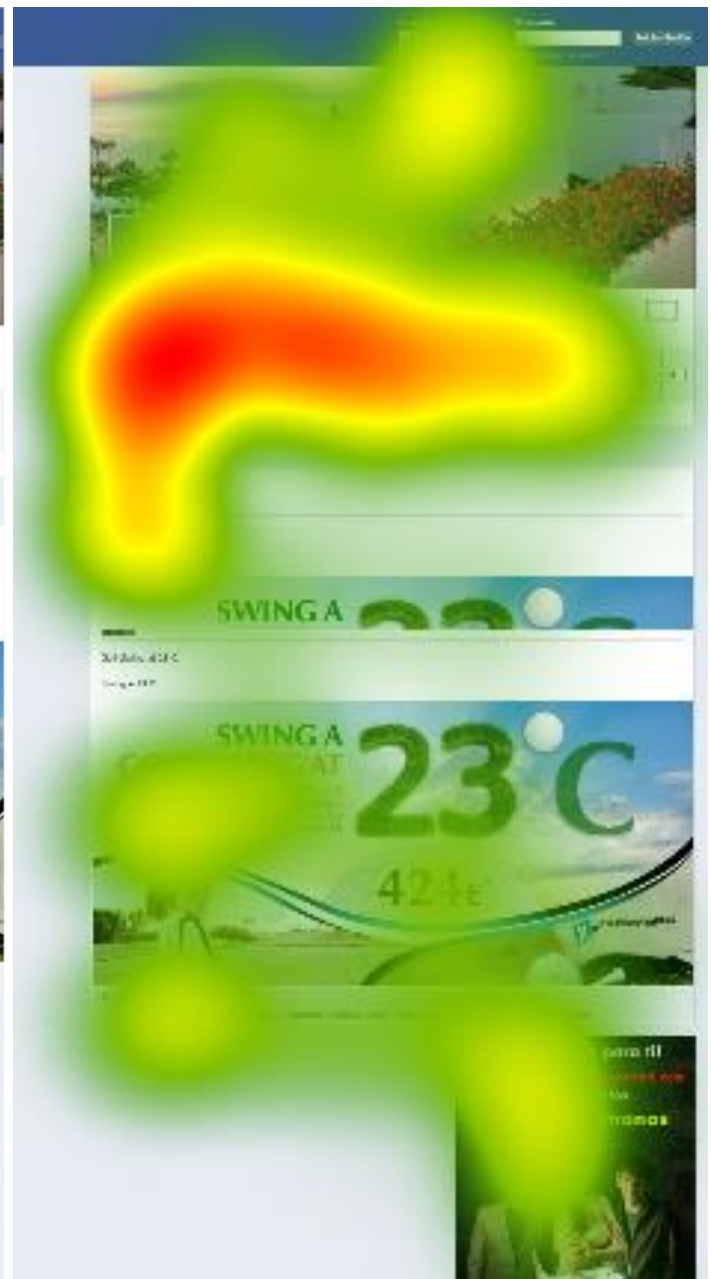

de consulta de disponibilidad también es una zona donde se concentran las primeras fijaciones.

A continuación los participantes dirigen su mirada a la derecha, donde se encuentra por un lado, la calificación que los viajeros le han dado al hotel y más abajo una lista de "Hoteles recomendados". Luego, éstos exploran la zona de la izquierda para leer los comentarios expuestos por otros viajeros y por último, dirigen de nuevo su vista a la parte de la derecha para fijarse en el contenido referente a otros hoteles de la zona ("Hoteles cerca de Adeje" y "Hoteles con alta valoración en o cerca de Adeje").

En este caso se comprueba que el área más caliente del mapa de calor se encuentra en la zona donde se encuentran los comentarios de otros viajeros. Esto es debido a que el objetivo principal cuando se entra a la página de Tripadvisor sea leer las experiencias de otros turistas, y en segundo lugar, porque uno de estos comentarios contenía la información necesaria para que los usuarios completaran su tarea.

En este caso el patrón de visualización que siguen los participantes vuelve a ser de izquierda a derecha, y éstos le prestan especial atención al área en el que se encuentran los comentarios de otros viajeros. Así mismo hay que destacar que en esta página los usuarios apenas se fijan en el banner y en la parte inferior de la pantalla (ver figura 5). 
Figura 5 - Gazeplot del sujeto más representativo y heatmap del perfil del hotel en Tripadvisor
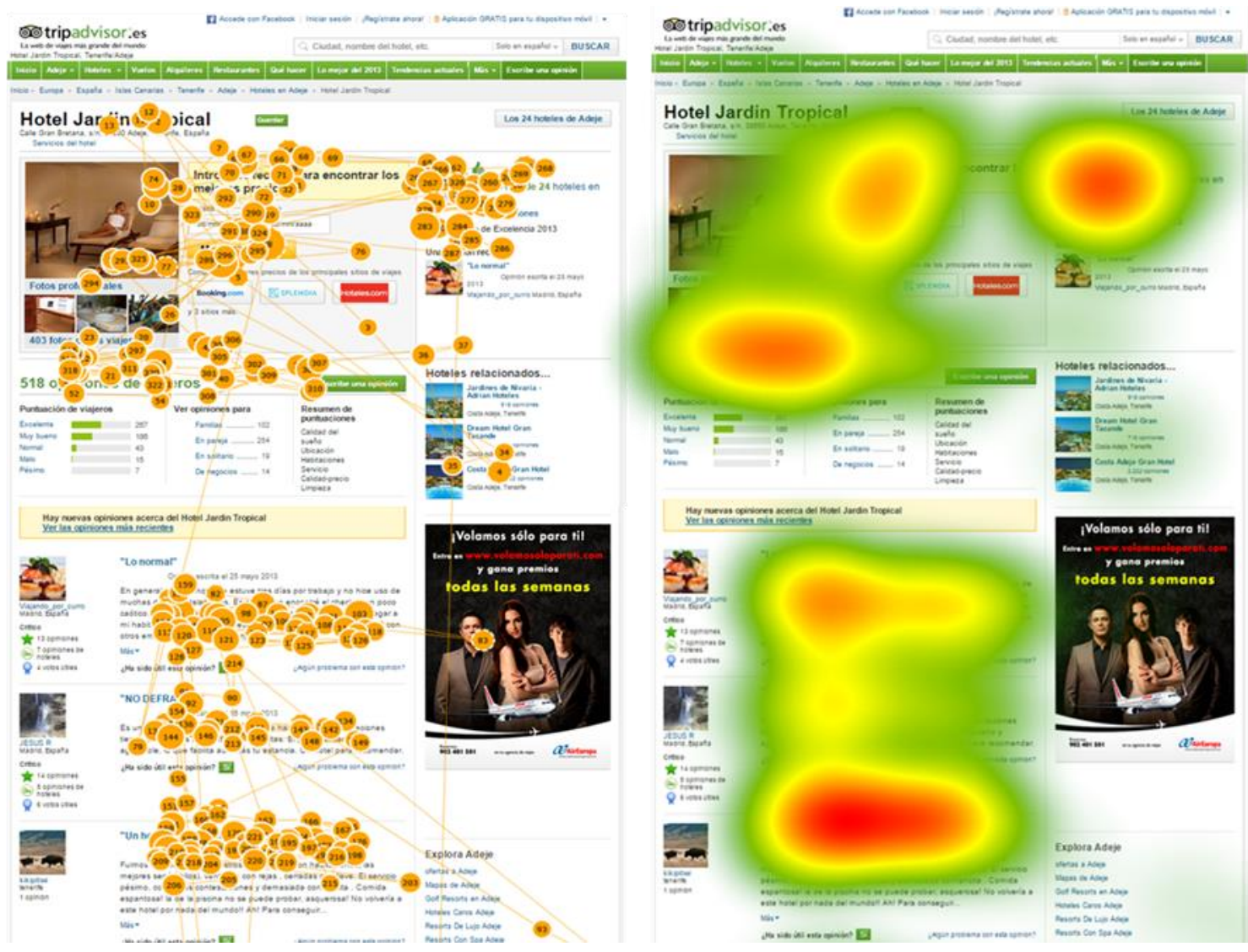

\subsection{Ceguera al banner}

En primer lugar, comprobamos si en nuestro estudio existió ceguera al banner de manera global (en las tres herramientas Travel 2.0). Los resultados del análisis de la fijación del participante muestran que el 95\% de los participantes se fijó al menos una vez en el banner localizado en el blog, un $98 \%$ de los participantes en el banner de Facebook, y un $67 \%$ de los participantes centraron su atención en el banner localizado en TripAdvisor. Aunque en niveles diferentes, podemos confirmar que la ceguera del banner no ocurre en los sitios web bajo estudio a partir de los datos de atención visual.

A continuación, comparamos el diferente grado de eficacia publicitaria de las herramientas Travel 2.0, en términos de atención dedicada al banner. Para responder a esta cuestión de investigación se aplicó un análisis de varianza (ANOVA) de un factor para diferentes variables métricas: tiempo hasta la primera fijación (Time to first fixation, TFF) y número de fijaciones antes (Fixations count before, FCB) de llegar al banner así como duración media de la fijación (Fixation Duration, FD) y número de fijaciones (Fixation Count, FC).

En este caso los resultados del análisis revelaron lo siguiente:

- Los participantes tardan menos tiempo en fijarse en el banner cuando visualizan la red social Facebook (14,05 seg.), seguido del blog (22,56 seg.), y por último cuando visitan Tripadvisor (38,68 seg.) (TFF: F-Snedecor = 19,100; d.f.1 = 2, d.f.2 = 154; sign.<,001). Los resultados demuestran que en el blog y en Tripadvisor se reconoce de alguna forma el anuncio con la visión periférica, y no le dedican ninguna fijación, o las fijaciones son escasas. Aunque sean valores medios, se

comprueba que la primera fijación en el banner no ocurre en los primeros movimientos oculares (como encontró Burke et al., 2005),

- La duración media de la fijación es mayor para el banner de la red Facebook $(0,22$ seg.), después para el del blog $(0,21 \mathrm{seg}$.), y por último para el de Tripadvisor (0,20 seg.) (FD: F-Snedecor = 3,068; d.f.1 = 2, d.f. $2=1,921$; sign. $=, 046)$.

- En el mismo orden anterior, los participantes se fijan más veces en el banner de Facebook (17), le sigue el del blog $(11,82)$, y en tercer lugar el de Tripadvisor $(5,90)$ (FC: FSnedecor = 15,058; d.f.1 = 2, d.f.2 = 154; sign. $<, 001$ ).

A modo de resumen podemos decir que los participantes no sólo dedican menos tiempo en visualizar el banner en Facebook sino que también realizan un mayor número de fijaciones en el banner y éstas tienen mayor duración. A continuación esta eficacia publicitaria se produce en el caso blog y por último, en el banner ubicado en la página de Tripavisor. La explicación puede venir del lado de la mayor simplicidad en el contenido editorial de los perfiles de Facebook de empresas.

Para comprobar si el nivel de atención al banner depende del perfil característico del sujeto se procedió a realizar sendos análisis de dependencia a partir de las variables consideradas independientes (género, edad y experiencia con las herramientas Travel 2.0).

Con respecto a la duración y el número de fijaciones según el género del sujeto se comprobó que no hay diferencias significativas en ningún caso. En este sentido tanto los hombres como las mujeres ignoran de la misma manera el banner, con 
valores similares para ambas variables ( $F D=210$ miliseg. en ambos sexos; $\mathrm{FC}_{\text {Hombre }}=10,00$ veces y $\mathrm{FC}_{\text {Mujer }}=10,88$ veces $)$.

En cuanto a la edad, la duración de la fijación es mayor para las personas menores de 35 años $(0,22 \mathrm{seg}$.) que para los mayores de esa edad $(0,20$ seg.) (FD: T-Student $=3,618$; d.f. $=1,922$; sign. $=, 000$ ).

Respecto a la experiencia con las herramientas Travel 2.0, la aplicación de un ANOVA mostró diferencias significativas a la hora de prestar atención al texto del banner entre los participantes más experimentados y los menos experimentados. En este caso los que usan las herramientas Travel 2.0. "algunas veces" $(0,22$ seg.) y "siempre que viajan" (0,21 seg.) tienen fijaciones más largas hacia el banner que los que las usan "rara vez" (0,20 seg.) (FD: FSnedecor $=3.675$; d.f. $=2,1,921$; sign. $=, 025$ ).

\subsection{Recuerdo del banner}

Con respecto al recuerdo auto-informado, los resultados revelaron que, de manera general, el $62 \%$ de los participantes recordaban haber visto algún anuncio durante su visita a alguna de las herramientas, y el 38\% restante no recordaba ninguno. En base a esto, quisimos profundizar sobre esta cuestión y los participantes que señalaron que recordaban algún anuncio tuvieron que responder a dos cuestiones específicas y en las que se medía el recuerdo espontáneo sobre el banner objeto de estudio.

En primer lugar, los sujetos tenían que indicar cuál era la marca/empresa del anuncio. Los resultados mostraron que más de la mitad de los participantes (55\%) no recordaban la marca o empresa del anuncio y sólo un $10 \%$ de los sujetos indicó que el anuncio hacía referencia a la compañía aérea (Air Europa). Otras respuestas de los participantes asociaron el anuncio a otras compañías (como lberia, Spanair, Booking,...), anuncio de coches de alquiler, de ofertas de otros hoteles, o de una compañía aérea sin especificar la marca.

En segundo lugar, el participante tenía que describir qué contenía la imagen del anuncio que había visualizado. Los resultados mostraron que el $28 \%$ de la muestra no recordaba la imagen y sólo un $3 \%$ recordaban con exactitud la composición de la misma, es decir, un fondo negro con los tres personajes famosos y la imagen de un avión superpuesta. Aunque un $10 \%$ de los participantes recordó el nombre de alguno de los famosos.

Otros participantes recordaban que el banner estaba formado por personas o parejas (30\%) sin aludir a un nombre concreto y referidas al anuncio de interés, otros recordaron que en el anuncio aparecía un avión (5\%), un 10\% de la muestra memorizó un campo de golf (presente en el perfil de Facebook) y una persona solo $(1,7 \%)$ hizo alusión al eslogan del anuncio aunque se equivocó en la expresión (viajeconnosotros.com). Se deduce de esta forma que los anuncios que recordaban también los participantes eran otros diferentes al banner de interés fundamental (como la promoción de campo de golf en Facebook, la imagen de una chica en el blog y en Tripadvisor, etc.).

Como complemento al análisis anterior se analizó el recuerdo sugerido entre los participantes para cuyo fin se les mostró un listado de empresas (Iberia, Air Europa y Booking) con un eslogan de una campaña reciente y se debía seleccionar la opción correcta. En este caso casi la mitad de los participantes (40\%) respondió que no sabían/no contestaba y un $37 \%$ de la muestra marcó la opción correcta.

\section{Discusión de resultados y recomendaciones}

Con el desarrollo de la Web 2.0, los turistas han adoptado un papel más activo a la hora de preparar sus viajes, y por otro lado, las empresas turísticas han tenido que aprovechar la oportunidad y adaptar sus estrategias de marketing a las nuevas herramientas Travel 2.0.

En la revisión de la literatura no encontramos estudios previos que analicen la eficacia publicitaria de un banner ubicado en diferentes sitios web o herramientas Web 2.0 en términos de atención, o sus resultados no son concluyentes. Además partimos del supuesto de que la mayoría de los trabajos analizados demuestran la ceguera al banner y por tanto, los usuarios los evitan o ignoran durante su visita a la web (Burke et al., 2005; Nielsen, 2007; Drèze \& Hussherr, 2003; Margarida, 2013).

Con respecto al primer objetivo de investigación, los resultados de nuestro estudio revelan que el patrón de visualización de los usuarios depende de la herramienta Travel 2.0 analizada. Al visualizar el blog del hotel y el perfil de Tripadvisor los usuarios siguen un patrón de visualización que va de izquierda a derecha (como resulta en los trabajos de Namah, 2001; Castelluccio, 2004; Djamasbi et al. 2010b) y le prestan especial atención al área que engloba el primer post (IAB Spain Research \& The Cocktail Analysis, 2009). En el caso de Tripadvisor los participantes le prestan especial atención al área en el que se encuentran los comentarios de otros viajeros.

En el caso de Facebook los navegantes de esta red social exploran la página con un movimiento de arriba hacia abajo y además éstos se fijan en mayor medida en el área superior izquierda de la pantalla tal y como se ha comprobado en la revisión de la literatura (Castellucio 2004; Djamasbi et al., 2010a; Mazman et al., 2010). Sin embargo, en el caso de Tripadvisor, los usuarios se fijan en menor medida en el banner y en la parte inferior de la pantalla (tal y como Nielsen, 2006; Shresta \& Lenz, 2007 descubren). Por el contrario, los usuarios del blog y de la red social se fijan bastante en la parte inferior y en el lado derecho de la misma, sobre todo cuando se ubican en estas zonas información de interés para el cliente potencial. Este resultado coincide con otros trabajos anteriores como el de Nielsen (2006) y Shresta and Lenz (2007).

Con respecto al segundo objetivo, en nuestro estudio se comprobó que un alto porcentaje de sujetos (más de las dos terceras partes) realizan al menos una fijación en el banner, siendo su duración media de 210 milisegundos. Por tanto, podemos confirmar que al contrario de lo que ocurre en otras investigaciones (e.g. Bayles, 2002; Burke et al., 2005; Nielsen, 2007; Drèze \& Hussher, 2003; Margarida, 2013) no existe ceguera del banner. Si bien los resultados revelan que los usuarios tardan cierto tiempo en prestar atención al banner. Esto puede deberse a que los usuarios reconocen el anuncio con la visión periférica, de ahí que la primera fijación en el mismo no ocurre en los primeros movimientos oculares (Burke et al., 2005).

Tras comprobar esta hipótesis general, se analizó si esta ausencia de eficacia publicitaria varía según diferentes variables de 
segmentación de usuario. Con respecto al género se comprobó que tanto los hombres como las mujeres ignoran de la misma manera el banner (como sostienen los trabajos de Drèze \& Hussherr, 2003; Margarida, 2013). Si tenemos en cuenta la edad, a diferencia de otros autores (e.g. Drèze \& Hussherr, 2003; Danaher et al., 2006), los resultados de nuestro estudio revelan que las personas menores de 35 años tienen fijaciones más largas hacia el banner que los mayores de 35 años. Por último, en el caso de la experiencia se determinó que los usuarios más experimentados con estas herramientas se fijan durante más tiempo en el banner que los que las usan escasamente. Estos resultados en cierta medida coincidentes con los trabajos de Thorbjornsen et al. (2002) y Crespo (2011).

Particularizando por tipo de herramienta, se ha comprobado que Facebook es la herramienta Travel 2.0 en la que se consigue una mayor eficacia publicitaria, seguida del blog y por último Tripadvisor. La razón a este hecho procede de la complejidad que el diseño de una página web (tamaño y formato del texto, inclusión de imágenes, etc.) puede tener sobre el patrón de visualización de los distintos sujetos que exploran las diferentes páginas (Djamasbi et al., 2011). En nuestro caso el perfil de Facebook mostró un menor contenido editorial que el resto. Además, si los usuarios tienen un mayor recuerdo/experiencia de cómo está estructurada una página Facebook, con respeto a un blog por ejemplo que no conocen, se amplificaría la ceguera al banner. En este sentido, la eficacia publicitaria de Facebook sería incluso más fuerte.

Con respecto al recuerdo del banner, algunos trabajos de investigación no alcanzan resultados concluyentes o algunos llegan a ser contradictorios. Aunque los datos de la memoria de reconocimiento no son especialmente malos, se comprueba que el $62 \%$ de los participantes recordaban algún anuncio durante su visita, y algo más de la mitad de la muestra no recordaba la marca/empresa del banner objeto de estudio. Además un 37\% de los usuarios marcó la opción correcta sobre la marca y el eslogan que componía el banner. Igualmente los participantes también recordaban otros anuncios diferentes al banner objeto de estudio que se encuentran presentes en otros lugares del escenario experimental.

En línea con lo que ocurre en otros estudios (Bayles, 2002; Drèze \& Hussherr, 2003; Burke et al., 2005) en nuestra investigación hay una disminución de la eficacia publicitaria en términos de recuerdo, con respecto a la atención visual. Esto nos sugiere que la utilización de ambas metodologías de investigación (observación a través de eye-tracking y recuerdo auto-informado) permite complementar y comprender mejor los resultados.

\section{Agradecimientos}

Este estudio ha recibido el apoyo financiero del Proyecto de Investigación Fundamental No Orientada (2012) del Ministerio de Economía y Competitividad español (Proyecto ECO2012-39576) y de la Junta de Andalucía (Proyecto SEJ-1980).

\section{Referencias}

Abuín, N. (2008). La publicidad en periódicos electrónicos: Creación y evaluación de un modelo de eficacia. Universidad Complutense de Madrid. Retrieved November, 13, 2014 From: http://eprints.ucm.es/7958/ .
Bayles, M. E. (2002). Designing online banner advertisements: Should we animate? In Proceedings of the SIGCHI Conference on Human Factors in Computing Systems, Minneapolis, MN, April 2002, ACM Press, New York, 363-368.

Buhalis, D. \& Law, R. (2008). Progress in information technology and tourism management: 20 years on and 10 years after the Internet - The state of eTourism research. Tourism Management, 29(4) 609-623.

Burke, M., Hornof, A., Nilsen, E. \& Gorman, N. (2005). High-cost banner blindness: Ads increase perceived workload, hinder visual search, and are forgotten. ACM Transactions Computer-Human Interaction, 12(4), 423-445.

Castelluccio, M. (2004). How We Read Web Pages. Strategic Finance 53 , Institute of Management Accountants Inc. Retrieved October, 31, 2010 http://www.highbeam.com/doc/1G1-127980114.html

Cheung, C.M., Chiu, P.Y. \& Lee, M.K. (2011). Online social networks: Why do students use facebook?. Computers in Human Behavior, 27(4), 1337-1343.

Chiou, W.B., Wan, C.S. \& Lee, H.Y. (2008).Virtual experience vs. brochures in the advertisement of scenic spots: How cognitive preferences and order effects influence advertising effects on consumers. Tourism Management, 29(1), 146-150.

Crespo, E. (2011). Eficacia de la promoción de ventas on-line. Influencia del tipo de incentivo promocional y la experiencia de uso web. Departamento de Comercialización e Investigación de Mercados. Universidad de Granada. Dahlen, M. (2001). Banner advertisements through a new lens. Journal of Advertising Research, 41(4), 23-30.

Danaher, P. J., Mullarkey, G. W., \& Essegaier, S. (2006). Factors affecting web site visit duration: A cross-domain analysis. Journal of Marketing Research, 43(2), 182-194.

Djamasbi, S., Siegel, M. \& Tullis, T. (2010a).Generation Y, web design, and eye tracking.International Journal of Human-Computer Studies, 68(5), 307-323.

Djamasbi, S., Siegel M., Tullis, T., \& Dai, R. (2010b). Efficiency, trust, and visual appeal: Usability testing through eye tracking. In System Sciences (HICSS), 2010 43rd Hawaii International Conference on IEEE, (pp. 1-10), IEE, Hawaii.

Djamasbi, S., Siegel M. \& Tullis, T. (2011). Visual hierarchy and viewing behavior: An eye tracking study. In Human-Computer Interaction. Design and Development Approaches, (pp. 331-340). Springer Berlin Heidelberg.

Drèze, X. \& Hussherr F.X. (2003). Internet advertising: is anybody watching? Journal of Interactive Marketing, 17(4), 8-23.

Gong, W. \&. Maddox, L.M. (2003). Measuring web advertising effectiveness in China.Journal of Advertising Research, 43(1), 34-49.

Goodrich, K. (2014). The gender gap: Brain- Processing sexes shape attitudes about online advertising. Journal of Advertising Research, 54(1), 61-72.

Hernández-Méndez, J. \& Muñoz-Leiva, F. (2015). What type of online advertising is most effective for eTourism 2.0? An eye tracking study based on the characteristics of tourists. Computers in Human Behavior, 50, 618-626.

Hernández-Méndez, J., Muñoz-Leiva, F., \& Sánchez-Fernández, J. (2015). The influence of e-word-of-mouth on travel decision-making: consumer profiles. Current issues in tourism, 18(11), 1001-1021.

Hollis, N. (2005). Ten years of learning on how online advertising builds brand. Journal of Advertising Research, 45, 255-268.

Hsieh, Y.C. \& Chen, K.H. (2011). How different information types affect viewer's attention on internet advertising. Computers in Human Behavior, 27(2), 935- 945.

IAB Spain Research \& The Cocktail Analysis (2009). Claves sobre la Interacción visual con la publicidad web. Aplicación de la técnica eye tracking. Retrieved November, 10, 2014 From: http://www.iabspain.net/.

International Telecommunication Union, ITU (2002). Methodology for the subjective assessment of the quality of television pictures. ITU-R BT.50011. Retrieved March, 12, 2012 From: http://www.itu.int/rec/R-RECBT.500/en .

León Sáez de Ybarra, J.L. (2009). Nuevos Soportes y mercados de la publicidad digital. Transiciones y experiencias. Pensar la Publicidad. Revista Internacional de Investigaciones Publicitarias, 3(2), 17-30.

Lindgaard, G., Fernandes, G., Dudek, C. \& Brown, J. (2006). Attention web designers: You have 50 milliseconds to make a good first impression! Behaviour \& Information Technology, 25(2), 115-126. 
Lohtia, R., Donthu, N. \& Yaveroglu, I. (2007). Evaluating the efficiency of Internet banner advertisements.Journal of Business Research, 60(4), 365-370.

Malhotra, N.K. (1997). Investigación de Mercados. Un enfoque práctico. 2a ed., Prentice Hall Hispanoamericana, México.

Margarida, A. (2013). Do users look at banner ads on Facebook? Journal of Research in Interactive Marketing, 7(2), 119-139.

Mazman, S. G., Akbal, S., Tüzün, H., \& Yeniad, M. (2010). Usability testing of departmental websites: A case study with authentic users and authentic tasks. Proccedings of ICERI2010 Conference, 15th-17th November 2010, Madrid.

Mendes-Filho, L. A. M., \& Tan, F. B. (2008). An overview on User-Generated Content and the empowerment of Online Travellers.FARN Journal, 7(2), 17-30.

Muñoz-Leiva, F., Hernández-Méndez, J. \& Sánchez-Fernández, J. (2012). Generalising user behaviour in online travel sites through the Travel 2.0 website acceptance model. Online Information Review, 36(6), 879-902.

Namahn (2001). Use eye tracking for usability testing. Research Document, TechGig, Bussels, 1-12.

Nielsen, J. (2006). F-shaped pattern for Reading web content. Retrieved November, 11, 2014 From: http://www.nngroup.com/articles/f-shapedpattern-reading-web-content/

Nielsen, J. (2007). Banner blindness: old and new findings. Retrieved November, 11, 2014 From: http://www.nngroup.com/articles/bannerblindness-old-and-new-findings/

Nielsen (2014). The digital consumer. Retrieved November, 11, 2014 From: http://www.nielsen.com/content/dam/corporate/us/en/reportsdownloads/2014\%20Reports/the-digital-consumer-report-feb-2014.pdf

Noton, D. \& Stark, L. (1971). Eye movements and visual perception. Scientific American, 224, 34-43.

Owens, J. W., Chaparro, B.S. \& Palmer, E.M. (2011). Text advertising blindness: the new banner blindness? Journal of Usability Studies, 6(3), 172-197.

Öz, M. (2015). Social media utilization of tourists for travel-related purposes. International Journal of Contemporary Hospitality Management, 27(5), 1003-1023.

Pan, B., \& Zhang, L. (2010). An eyetracking study on online hotel decisionmaking: The effects of images and number of options. Travel and TourismResearch, Association Annual Conference, San Antonio, June 20-22.

Rayner, K. (1998). Eye movements in Reading and information processing: 20 years of Research. Psychological Bulletin, 124(3), 372-422.

Russo, J. E. (1978). Eye fixations can save the World: A critical evaluation and a comparison between eye fixation and other information processing methodologies. Advances in Consumer Research, 5(1), 561-570.

San José Cabezudo, R., Gutiérrez, A.M., \& Gutiérrez, J. (2004). Determinantes de la eficacia publicitaria del sitio web. Una aplicación del ELM. Revista Española de Investigación de Marketing. ESIC, 8(2), 93-121.

Shrestha, S. \& Lenz, K. (2007). Eye gaze patterns while searching vs. browsing a Website. Usability News, 9(1), 1-9.

Thorbjørnsen, H., Supphellen, M., Nysveen, H., \& Egil, P. (2002). Building brand relationships online: a comparison of two interactive applications. Journal of interactive marketing, 16(3), 17-34.

Van Trijp, H.C. (2009). Consumer understanding and nutritional communication: Key issues in the context of the new EU legislation. European Journal of Nutrition, 48, 1, 41-48.

Wedel, M. \& Pieters, R. (2008). A Review of Eye-Tracking Research in Marketing, in Naresh K. Malhotra (ed.), Review of Marketing Research, vol. 4 (pp.123 - 147), Emerald Group Publishing Limited, Armonk, NY (USA).

Yaveroglu, I. \& Donthu, N. (2008). Advertising repetition and placement issues in on-line environments. Journal of Advertising, 37(2), 31-43.

Zikmund, William G. (2003). Fundamentos de investigación de mercados. Thomson, Madrid.

Recibido: 29.10.2015

Reenviado: 04.07. 2016

Aceptado: 06.07.2016 